\title{
FDM for the integral-differential equation of the hyperbolic type
}

\section{Zilal Direk and Maksat Ashyraliyev²}

\section{${ }^{*}$ Correspondence:}

maksat.ashyralyyev@bahcesehir. edu.tr

${ }^{2}$ Department of Mathematics and Computer Sciences, Bahcesehir University, Besiktas, Istanbul, 34353, Turkey

Full list of author information is available at the end of the article

\begin{abstract}
In this paper, the second order of accuracy difference scheme approximately solving the initial value problem for an integral-differential equation of the hyperbolic type in a Hilbert space $H$ is presented. The stability estimates for the solution of this difference scheme are obtained. Theoretical results are supported by numerical examples.
\end{abstract}

PACS Codes: $02.60 . \mathrm{Lj} ; 02.60 . \mathrm{Nm} ; 02.70 . \mathrm{Bf} ; 87.10 . \mathrm{Ed}$

Keywords: finite difference method; integral-differential equation of the hyperbolic type

\section{Introduction}

We consider the initial value problem

$$
\left\{\begin{array}{l}
\frac{d^{2} u(t)}{d t^{2}}+A u(t)=\int_{-t}^{t} B(\rho) u(\rho) d \rho+f(t), \quad-1 \leq t \leq 1, \\
u(0)=u_{0}, \quad u^{\prime}(0)=u_{0}^{\prime}
\end{array}\right.
$$

for an integral-differential equation in a Hilbert space $H$ with unbounded linear operators $A$ and $B(t)$ in $H$ with dense domain $D(A) \subset D(B(t))$ and

$$
\left\|B(t) A^{-1}\right\|_{H \rightarrow H} \leq M, \quad-1 \leq t \leq 1 .
$$

It is well known that various initial-boundary value problems for the integral-differential equation of the hyperbolic type with two dependent limits can be reduced to the initial value problem (1) in a Hilbert space $H$; see [1-3].

A function $u(t)$ is called a solution of the problem (1) if the following conditions are satisfied:

(i) $u(t)$ is twice continuously differentiable on $[-1,1]$. The derivative at the endpoints of the segment are understood as the appropriate unilateral derivatives.

(ii) The element $u(t)$ belongs to $D(A)$ for all $t \in[-1,1]$, and the function $A u(t)$ is continuous on $[-1,1]$.

(iii) $u(t)$ satisfies the equations and the initial conditions (1).

A solution of the problem (1) defined in this manner will from now on be referred to as a solution of the problem (1) in the space $C(H)=C([-1,1], H)$ of all continuous functions $\varphi(t)$ defined on $[-1,1]$ with values in $H$ equipped with the norm

$$
\|\varphi\|_{C(H)}=\max _{-1 \leq t \leq 1}\|\varphi(t)\|_{H} .
$$

@2014Direk and Ashyraliyev; licensee Springer. This is an Open Access article distributed under the terms of the Creative Commons Attribution License (http://creativecommons.org/licenses/by/2.0), which permits unrestricted use, distribution, and reproduction in any medium, provided the original work is properly cited. 
We consider the problem (1) under the assumption that $A$ is a positive definite self-adjoint operator with $A \geq \delta I$, where $\delta>\delta_{0}>0$.

Theorem 1 [4] Suppose that $u_{0} \in D(A), u_{0}^{\prime} \in D\left(A^{1 / 2}\right)$ and $f(t)$ is a continuously differentiable function on $[-1,1]$. Then there is a unique solution of the problem (1) and the stability inequalities

$$
\begin{aligned}
& \max _{-1 \leq t \leq 1}\left\|\frac{d^{2} u(t)}{d t^{2}}\right\|_{H}+\max _{-1 \leq t \leq 1}\|A u(t)\|_{H} \\
& \quad \leq M^{*}\left[\left\|A u_{0}\right\|_{H}+\left\|A^{1 / 2} u_{0}^{\prime}\right\|_{H}+\|f(0)\|_{H}+\int_{-1}^{1}\left\|f^{\prime}(s)\right\|_{H} d s\right]
\end{aligned}
$$

hold, where $M^{*}$ does not depend on $u_{0}, u_{0}^{\prime}$, and $f(t), t \in[-1,1]$.

In [4] the first order of accuracy difference scheme

$$
\left\{\begin{array}{l}
\frac{u_{k+1}-2 u_{k}+u_{k-1}}{\tau^{2}}+A u_{k+1}=\sum_{j=-k}^{k} B_{j} u_{j} \tau+f_{k}, \quad k=1, \ldots, N-1, \\
\frac{u_{k+1}-2 u_{k}+u_{k-1}}{\tau^{2}}+A u_{k-1}=-\sum_{j=k}^{-k} B_{j} u_{j} \tau+f_{k}, \quad k=-N+1, \ldots, 0, \\
\tau=\frac{1}{N}, \quad B_{k}=B\left(t_{k}\right), \quad f_{k}=f\left(t_{k}\right), \quad t_{k}=k \tau, \quad k=-N, \ldots, N, \\
u_{0}=u(0), \quad\left(I+\tau^{2} A\right) \frac{u_{1}-u_{0}}{\tau}=u_{0}^{\prime}
\end{array}\right.
$$

for approximate solutions of the problem (1) was considered.

Theorem 2 [4] Suppose that the requirements of Theorem 1 are satisfied. Then for the solution of difference scheme (4) the stability inequalities

$$
\begin{aligned}
& \max _{-N+1 \leq k \leq N-1}\left\|\frac{u_{k+1}-2 u_{k}+u_{k-1}}{\tau^{2}}\right\|_{H}+\max _{-N \leq k \leq N}\left\|A u_{k}\right\|_{H} \\
& \leq M^{*}\left[\left\|A u_{0}\right\|_{H}+\left\|A^{1 / 2} u_{0}^{\prime}\right\|_{H}+\left\|f_{0}\right\|_{H}+\sum_{k=-N+1}^{N}\left\|f_{k}-f_{k-1}\right\|_{H}\right]
\end{aligned}
$$

hold, where $M^{*}$ does not depend on $u_{0}^{\prime}, u_{0}$, and $f_{k}, k=-N, \ldots, N$.

In this paper, we consider the second order of accuracy difference scheme

$$
\left\{\begin{array}{l}
\frac{u_{k+1}-2 u_{k}+u_{k-1}}{\tau^{2}}+\frac{1}{2} A u_{k}+\frac{1}{4} A\left(u_{k+1}+u_{k-1}\right) \\
\quad=\tau \sum_{j=-k+1}^{k} B_{j-\frac{1}{2}}\left(\frac{u_{j}+u_{j-1}}{2}\right)+f_{k}, \quad k=1, \ldots, N-1, \\
\frac{u_{k+1}-2 u_{k}+u_{k-1}}{\tau^{2}}+\frac{1}{2} A u_{k}+\frac{1}{4} A\left(u_{k+1}+u_{k-1}\right) \\
\quad=-\tau \sum_{j=k+1}^{-k} B_{j-\frac{1}{2}}\left(\frac{u_{j}+u_{j-1}}{2}\right)+f_{k}, \quad k=-N+1, \ldots,-1, \\
\tau=\frac{1}{N}, \quad f_{k}=f\left(t_{k}\right), \quad t_{k}=k \tau, \quad k=-N, \ldots, N, \\
B_{k-\frac{1}{2}}=B\left(t_{k}-\frac{\tau}{2}\right), \quad k=-N+1, \ldots, N, \\
u(0)=u_{0}, \quad\left(I+\tau^{2} A\right)\left(\frac{u_{1}-u_{0}}{\tau}\right)=\frac{\tau}{2}\left(f_{0}-A u_{0}\right)+u_{0}^{\prime}, \\
\left(I+\tau^{2} A\right)\left(\frac{u_{0}-u_{-1}}{\tau}\right)=\frac{\tau}{2}\left(A u_{0}-f_{0}\right)+u_{0}^{\prime},
\end{array}\right.
$$

for approximate solutions of the problem (1). The paper is organized as follows. In Section 2 we obtain the stability estimates for the solution of difference scheme (6). Numerical 
illustrations for the simple test problem are provided in Section 3. The paper is concluded with remarks in Section 4.

\section{The stability estimates for the solution of difference scheme}

Theorem 3 Suppose that the requirements of Theorem 1 are satisfied. Then for the solution of difference scheme (6) the stability inequalities

$$
\begin{gathered}
\max _{-N+1 \leq k \leq N-1}\left\|\frac{u_{k+1}-2 u_{k}+u_{k-1}}{\tau^{2}}\right\|_{H}+\max _{-N+1 \leq k \leq N}\left\|\frac{A\left(u_{k}+u_{k-1}\right)}{2}\right\|_{H} \\
\leq M^{*}\left[\left\|A u_{0}\right\|_{H}+\left\|A^{1 / 2} u_{0}^{\prime}\right\|_{H}+\left\|f_{0}\right\|_{H}+\sum_{k=-N+1}^{N}\left\|f_{k}-f_{k-1}\right\|_{H}\right]
\end{gathered}
$$

hold, where $M^{*}$ does not depend on $u_{0}^{\prime}, u_{0}$, and $f_{k}, k=-N, \ldots, N$.

Proof By [5], the second order of accuracy difference scheme

$$
\left\{\begin{array}{l}
\frac{u_{k+1}-2 u_{k}+u_{k-1}}{\tau^{2}}+\frac{1}{2} A u_{k}+\frac{1}{4} A\left(u_{k+1}+u_{k-1}\right)=\psi_{k}, \quad k=1, \ldots, N-1, \\
\left(I+\tau^{2} A\right) \frac{u_{1}-u_{0}}{\tau}=\frac{\tau}{2}\left(\psi_{0}-A u_{0}\right)+u_{0}^{\prime}, \quad u_{0}=u(0)
\end{array}\right.
$$

has the solution

$$
\begin{aligned}
u_{1}= & \left(I+\tau^{2} A\right)^{-1}\left[\left(I+\frac{\tau^{2}}{2} A\right) u_{0}+\tau u_{0}^{\prime}+\frac{\tau^{2}}{2} \psi_{0}\right], \\
u_{k}= & {\left[R^{k}+\frac{1}{2 i} A^{-1 / 2}\left(I-\frac{i \tau A^{1 / 2}}{2}\right)\left(R^{k}-\tilde{R}^{k}\right)\right.} \\
& \left.\times\left\{\left(I+\frac{i \tau A^{1 / 2}}{2}\right) \frac{\tau}{2} A-i A^{1 / 2}\left(I+\tau^{2} A\right)\right\}\left(I+\tau^{2} A\right)^{-1}\right] u_{0} \\
& +\frac{i}{2} A^{-1 / 2}\left(I-\frac{i \tau A^{1 / 2}}{2}\right)\left(R^{k}-\tilde{R}^{k}\right)\left(I+\tau^{2} A\right)^{-1}\left(I+\frac{i \tau A^{1 / 2}}{2}\right) u_{0}^{\prime} \\
& +\frac{i}{2} A^{-1 / 2}\left(I-\frac{i \tau A^{1 / 2}}{2}\right)\left(R^{k}-\tilde{R}^{k}\right)\left(I+\tau^{2} A\right)^{-1}\left(I+\frac{i \tau A^{1 / 2}}{2}\right) \frac{\tau}{2} \psi_{0} \\
& -\sum_{j=1}^{k-1} \frac{\tau}{2 i} A^{-1 / 2}\left(R^{k-j}-\tilde{R}^{k-j}\right) \psi_{j}, \quad k=2, \ldots, N,
\end{aligned}
$$

where $R=\left(I-\frac{i \tau A^{1 / 2}}{2}\right)\left(I+\frac{i \tau A^{1 / 2}}{2}\right)^{-1}, \tilde{R}=\left(I+\frac{i \tau A^{1 / 2}}{2}\right)\left(I-\frac{i \tau A^{1 / 2}}{2}\right)^{-1}$. By putting $\psi_{0}=f_{0}, \psi_{k}=$ $\tau \sum_{s=-k+1}^{k} B_{s-\frac{1}{2}}\left(\frac{u_{s}+u_{s-1}}{2}\right)+f_{k}, k=1, \ldots, N-1$, we obtain

$$
\begin{aligned}
A u_{k}= & {\left[R^{k}+\frac{1}{2 i} A^{-1 / 2}\left(I-\frac{i \tau A^{1 / 2}}{2}\right)\left(R^{k}-\tilde{R}^{k}\right)\right.} \\
& \left.\times\left\{\left(I+\frac{i \tau A^{1 / 2}}{2}\right) \frac{\tau}{2} A-i A^{1 / 2}\left(I+\tau^{2} A\right)\right\}\left(I+\tau^{2} A\right)^{-1}\right] A u_{0} \\
& +\frac{i}{2} A^{1 / 2}\left(I-\frac{i \tau A^{1 / 2}}{2}\right)\left(R^{k}-\tilde{R}^{k}\right)\left(I+\tau^{2} A\right)^{-1}\left(I+\frac{i \tau A^{1 / 2}}{2}\right) u_{0}^{\prime} \\
& +\frac{i}{2} A^{1 / 2}\left(I-\frac{i \tau A^{1 / 2}}{2}\right)\left(R^{k}-\tilde{R}^{k}\right)\left(I+\tau^{2} A\right)^{-1}\left(I+\frac{i \tau A^{1 / 2}}{2}\right) \frac{\tau}{2} f_{0}
\end{aligned}
$$




$$
\begin{aligned}
& -\sum_{j=1}^{k-1} \frac{\tau^{2}}{2 i} A^{1 / 2}\left(R^{k-j}-\tilde{R}^{k-j}\right) \sum_{s=-j+1}^{j} B_{s-\frac{1}{2}}\left(\frac{u_{s}+u_{s-1}}{2}\right) \\
& -\sum_{j=1}^{k-1} \frac{\tau A^{1 / 2}}{2 i}\left(R^{k-j}-\tilde{R}^{k-j}\right) f_{j}, \quad k=2, \ldots, N .
\end{aligned}
$$

Since $i \tau A^{1 / 2}=\left(I-\frac{i \tau A^{1 / 2}}{2}\right)(\tilde{R}-I)=\left(I+\frac{i \tau A^{1 / 2}}{2}\right)(I-R)$, we have

$$
\begin{aligned}
& \sum_{j=s}^{k-1} \frac{\tau A^{1 / 2}}{2 i}\left(R^{k-j}-\tilde{R}^{k-j}\right)=\frac{1}{2}\left(I-\frac{i \tau A^{1 / 2}}{2}\right) R^{k-s}+\frac{1}{2}\left(I+\frac{i \tau A^{1 / 2}}{2}\right) \tilde{R}^{k-s}-I, \\
& \sum_{j=-s+1}^{k-1} \frac{\tau A^{1 / 2}}{2 i}\left(R^{k-j}-\tilde{R}^{k-j}\right)=\frac{1}{2}\left(I+\frac{i \tau A^{1 / 2}}{2}\right) R^{k+s}+\frac{1}{2}\left(I-\frac{i \tau A^{1 / 2}}{2}\right) \tilde{R}^{k+s}-I .
\end{aligned}
$$

Thus,

$$
\begin{aligned}
\sum_{j=1}^{k-1} & \frac{\tau^{2} A^{1 / 2}}{2 i}\left(R^{k-j}-\tilde{R}^{k-j}\right) \sum_{s=-j+1}^{j} B_{s-\frac{1}{2}}\left(\frac{u_{s}+u_{s-1}}{2}\right) \\
= & \sum_{s=1}^{k-1} \sum_{j=s}^{k-1} \frac{\tau A^{1 / 2}}{2 i}\left(R^{k-j}-\tilde{R}^{k-j}\right) B_{s-\frac{1}{2}}\left(\frac{u_{s}+u_{s-1}}{2}\right) \tau \\
& +\sum_{s=-k+2}^{0} \sum_{j=-s+1}^{k-1} \frac{\tau A^{1 / 2}}{2 i}\left(R^{k-j}-\tilde{R}^{k-j}\right) B_{s-\frac{1}{2}}\left(\frac{u_{s}+u_{s-1}}{2}\right) \tau \\
= & \frac{1}{2} \sum_{s=1}^{k-1}\left[\left(I-\frac{i \tau A^{1 / 2}}{2}\right) R^{k-s}+\left(I+\frac{i \tau A^{1 / 2}}{2}\right) \tilde{R}^{k-s}-2 I\right] B_{s-\frac{1}{2}}\left(\frac{u_{s}+u_{s-1}}{2}\right) \tau \\
& +\frac{1}{2} \sum_{s=-k+2}^{0}\left[\left(I+\frac{i \tau A^{1 / 2}}{2}\right) R^{k+s}+\left(I-\frac{i \tau A^{1 / 2}}{2}\right) \tilde{R}^{k+s}-2 I\right] B_{s-\frac{1}{2}}\left(\frac{u_{s}+u_{s-1}}{2}\right) \tau
\end{aligned}
$$

Furthermore,

$$
\begin{aligned}
\sum_{j=1}^{k-1} & \frac{\tau A^{1 / 2}}{2 i}\left(R^{k-j}-\tilde{R}^{k-j}\right) f_{j} \\
= & -f_{k-1}+\frac{1}{2}\left[\left(I-\frac{i \tau A^{1 / 2}}{2}\right) R^{k-1}+\left(I+\frac{i \tau A^{1 / 2}}{2}\right) \tilde{R}^{k-1}\right] f_{0} \\
& +\frac{1}{2} \sum_{j=1}^{k-1}\left[\left(I-\frac{i \tau A^{1 / 2}}{2}\right) R^{k-j}+\left(I+\frac{i \tau A^{1 / 2}}{2}\right) \tilde{R}^{k-j}\right]\left(f_{j}-f_{j-1}\right) .
\end{aligned}
$$

Putting (10)-(11) in (9), we get

$$
\begin{aligned}
A u_{k}= & {\left[R^{k}+\frac{1}{2 i} A^{-1 / 2}\left(I-\frac{i \tau A^{1 / 2}}{2}\right)\left(R^{k}-\tilde{R}^{k}\right)\right.} \\
& \left.\times\left\{\left(I+\frac{i \tau A^{1 / 2}}{2}\right) \frac{\tau}{2} A-i A^{1 / 2}\left(I+\tau^{2} A\right)\right\}\left(I+\tau^{2} A\right)^{-1}\right] A u_{0}
\end{aligned}
$$




$$
\begin{aligned}
& +\frac{i}{2} A^{1 / 2}\left(I-\frac{i \tau A^{1 / 2}}{2}\right)\left(R^{k}-\tilde{R}^{k}\right)\left(I+\tau^{2} A\right)^{-1}\left(I+\frac{i \tau A^{1 / 2}}{2}\right) u_{0}^{\prime} \\
& +\frac{i}{2} A^{1 / 2}\left(I-\frac{i \tau A^{1 / 2}}{2}\right)\left(R^{k}-\tilde{R}^{k}\right)\left(I+\tau^{2} A\right)^{-1}\left(I+\frac{i \tau A^{1 / 2}}{2}\right) \frac{\tau}{2} f_{0} \\
& +f_{k-1}-\frac{1}{2}\left[\left(I-\frac{i \tau A^{1 / 2}}{2}\right) R^{k-1}+\left(I+\frac{i \tau A^{1 / 2}}{2}\right) \tilde{R}^{k-1}\right] f_{0} \\
& -\frac{1}{2} \sum_{j=1}^{k-1}\left[\left(I-\frac{i \tau A^{1 / 2}}{2}\right) R^{k-j}+\left(I+\frac{i \tau A^{1 / 2}}{2}\right) \tilde{R}^{k-j}\right]\left(f_{j}-f_{j-1}\right) \\
& +\frac{1}{2} \sum_{s=1}^{k-1}\left[2 I-\left(I-\frac{i \tau A^{1 / 2}}{2}\right) R^{k-s}-\left(I+\frac{i \tau A^{1 / 2}}{2}\right) \tilde{R}^{k-s}\right] B_{s-\frac{1}{2}}\left(\frac{u_{s}+u_{s-1}}{2}\right) \tau \\
& +\frac{1}{2} \sum_{s=-k+2}^{0}\left[2 I-\left(I+\frac{i \tau A^{1 / 2}}{2}\right) R^{k+s}-\left(I-\frac{i \tau A^{1 / 2}}{2}\right) \tilde{R}^{k+s}\right] B_{s-\frac{1}{2}}\left(\frac{u_{s}+u_{s-1}}{2}\right) \tau \\
& k=2, \ldots, N .
\end{aligned}
$$

Using $I+R^{-1}=2\left(I-\frac{i \tau A^{1 / 2}}{2}\right)^{-1}$ and $I+\tilde{R}^{-1}=2\left(I+\frac{i \tau A^{1 / 2}}{2}\right)^{-1}$, from (12) we obtain

$$
\begin{aligned}
\frac{A\left(u_{k}+u_{k-1}\right)}{2}= & {\left[R^{k}\left(I-\frac{i \tau A^{1 / 2}}{2}\right)^{-1}+\frac{A^{-1 / 2}}{2 i}\left(R^{k}-\tilde{R}^{k-1}\right)\right.} \\
& \left.\times\left\{\left(I+\frac{i \tau A^{1 / 2}}{2}\right) \frac{\tau A}{2}-i A^{1 / 2}\left(I+\tau^{2} A\right)\right\}\left(I+\tau^{2} A\right)^{-1}\right] A u_{0} \\
& +\frac{i A^{1 / 2}}{2}\left(R^{k}-\tilde{R}^{k-1}\right)\left(I+\tau^{2} A\right)^{-1}\left(I+\frac{i \tau A^{1 / 2}}{2}\right) u_{0}^{\prime} \\
& +\frac{i A^{1 / 2}}{2}\left(R^{k}-\tilde{R}^{k-1}\right)\left(I+\tau^{2} A\right)^{-1}\left(I+\frac{i \tau A^{1 / 2}}{2}\right) \frac{\tau}{2} f_{0} \\
& +\left(I-\frac{R^{k-1}+\tilde{R}^{k-1}}{2}\right) f_{0}+\sum_{j=1}^{k-1}\left(I-\frac{R^{k-j}+\tilde{R}^{k-j}}{2}\right)\left(f_{j}-f_{j-1}\right) \\
& +\sum_{s=1}^{k-1}\left(I-\frac{R^{k-s}+\tilde{R}^{k-s}}{2}\right) B_{s-\frac{1}{2}}\left(\frac{u_{s}+u_{s-1}}{2}\right) \tau \\
& +\sum_{s=-k+1}^{0}\left(I-\frac{R^{k+s-1}+\tilde{R}^{k+s-1}}{2}\right) B_{s-\frac{1}{2}}\left(\frac{u_{s}+u_{s-1}}{2}\right) \tau \\
& k=2, \ldots, N .
\end{aligned}
$$

Then, using (2) and the following estimates:

$$
\begin{aligned}
& \|R\|_{H \rightarrow H} \leq 1, \quad\|\tilde{R}\|_{H \rightarrow H} \leq 1, \quad\left\|\left(I \pm \frac{i \tau A^{1 / 2}}{2}\right)^{-1}\right\|_{H \rightarrow H} \leq 1, \\
& \left\|\left(I \pm i \tau A^{1 / 2}\right)^{-1}\right\|_{H \rightarrow H} \leq 1, \quad\left\|\tau A^{1 / 2}\left(I \pm i \tau A^{1 / 2}\right)^{-1}\right\|_{H \rightarrow H} \leq 1
\end{aligned}
$$


yields

$$
\begin{aligned}
\left\|\frac{A\left(u_{k}+u_{k-1}\right)}{2}\right\|_{H} \leq & \frac{5}{2}\left\|A u_{0}\right\|_{H}+\left\|A^{1 / 2} u_{0}^{\prime}\right\|_{H}+\frac{5}{2}\left\|f_{0}\right\|_{H} \\
& +2 \sum_{j=-N+1}^{N}\left\|f_{j}-f_{j-1}\right\|_{H}+2 M \tau \sum_{j=-k+1}^{k-1}\left\|\frac{A\left(u_{j}+u_{j-1}\right)}{2}\right\|_{H},
\end{aligned}
$$

where $k=2, \ldots, N$. Furthermore, we have

$$
\frac{A\left(u_{1}+u_{0}\right)}{2}=\left[I+\left(I+\tau^{2} A\right)^{-1}\left(I+\frac{\tau^{2} A}{2}\right)\right] \frac{A u_{0}}{2}+\frac{\tau A}{2}\left(I+\tau^{2} A\right)^{-1} u_{0}^{\prime}+\frac{\tau^{2} A}{4}\left(I+\tau^{2} A\right)^{-1} f_{0},
$$

which gives us

$$
\left\|\frac{A\left(u_{1}+u_{0}\right)}{2}\right\|_{H} \leq\left\|A u_{0}\right\|_{H}+\frac{1}{2}\left\|A^{1 / 2} u_{0}^{\prime}\right\|_{H}+\frac{1}{4}\left\|f_{0}\right\|_{H} .
$$

In a similar way, one can prove that

$$
\begin{aligned}
\left\|\frac{A\left(u_{k}+u_{k-1}\right)}{2}\right\|_{H} \leq & \frac{5}{2}\left\|A u_{0}\right\|_{H}+\left\|A^{1 / 2} u_{0}^{\prime}\right\|_{H}+\frac{5}{2}\left\|f_{0}\right\|_{H} \\
& +2 \sum_{j=-N+1}^{N}\left\|f_{j}-f_{j-1}\right\|_{H}+2 M \tau \sum_{j=k+1}^{-k-1}\left\|\frac{A\left(u_{j}+u_{j-1}\right)}{2}\right\|_{H}
\end{aligned}
$$

holds for $k=-N+1, \ldots,-1$ and

$$
\left\|\frac{A\left(u_{0}+u_{-1}\right)}{2}\right\|_{H} \leq\left\|A u_{0}\right\|_{H}+\frac{1}{2}\left\|A^{1 / 2} u_{0}^{\prime}\right\|_{H}+\frac{1}{4}\left\|f_{0}\right\|_{H} .
$$

Using (14)-(17) and the theorem about the discrete analog of a Gronwall type integral inequality with two dependent limits $[4,6]$, we obtain

$$
\begin{aligned}
& \max _{-N+1 \leq k \leq N}\left\|\frac{A\left(u_{k}+u_{k-1}\right)}{2}\right\|_{H} \\
& \leq \tilde{M}\left[\left\|A u_{0}\right\|_{H}+\left\|A^{1 / 2} u_{0}^{\prime}\right\|_{H}+\left\|f_{0}\right\|_{H}+\sum_{k=-N+1}^{N}\left\|f_{k}-f_{k-1}\right\|_{H}\right] .
\end{aligned}
$$

Finally, using the triangle inequality in (6) we complete the proof of the estimates (7).

\section{Numerical example}

We consider the initial-boundary value problem

$$
\left\{\begin{array}{l}
\frac{\partial^{2} u(t, x)}{\partial t^{2}}-\frac{\partial^{2} u(t, x)}{\partial x^{2}}=\int_{-t}^{t} \frac{\partial^{2} u(s, x)}{\partial x^{2}} d s+\left(\frac{2 t^{3}}{3}+t^{2}+2\right) \sin x, \quad-1 \leq t \leq 1,0<x<\pi \\
u(0, x)=0, \quad u_{t}(0, x)=0, \quad 0 \leq x \leq \pi, \\
u(t, 0)=0, \quad u(t, \pi)=0, \quad-1 \leq t \leq 1,
\end{array}\right.
$$


Table 1 The errors between the exact solution of the problem (19) and the numerical solutions computed by using the first order and the second order of accuracy difference schemes (20) and (21), respectively

\begin{tabular}{llll}
\hline & $\mathbf{N}=\boldsymbol{M}=\mathbf{1 6}$ & $\mathbf{N}=\boldsymbol{M}=\mathbf{3 2}$ & $\mathbf{N =} \boldsymbol{M = 6 4}$ \\
\hline First order of accuracy difference scheme (20) & 0.0765 & 0.0384 & 0.0192 \\
Second order of accuracy difference scheme (21) & 0.0016 & 0.0004 & 0.0001 \\
\hline
\end{tabular}

which has the exact solution $u(t, x)=t^{2} \sin x$. Applying the first order of accuracy difference scheme (4) to the problem (19) yields

$$
\left\{\begin{array}{l}
\frac{u_{n}^{k+1}-2 u_{n}^{k}+u_{n}^{k-1}}{\tau^{2}}-\frac{u_{n+1}^{k+1}-2 u_{n}^{k+1}+u_{n-1}^{k+1}}{h^{2}}=\tau \sum_{i=-k}^{k} \frac{u_{n+1}^{i}-2 u_{n}^{i}+u_{n-1}^{i}}{h^{2}}+\left(\frac{2 t_{k}^{3}}{3}+t_{k}^{2}+2\right) \sin x_{n}, \\
n=1, \ldots, M-1, k=1, \ldots, N-1, \\
\frac{u_{n}^{k+1}-2 u_{n}^{k}+u_{n}^{k-1}}{\tau^{2}}-\frac{u_{n+1}^{k-1}-2 u_{n}^{k-1}+u_{n-1}^{k-1}}{h^{2}}=-\tau \sum_{i=k}^{-k} \frac{u_{n+1}^{i}-2 u_{n}^{i}+u_{n-1}^{i}}{h^{2}}+\left(\frac{2 t_{k}^{3}}{3}+t_{k}^{2}+2\right) \sin x_{n}, \\
n=1, \ldots, M-1, k=-N+1, \ldots, 0, \\
\tau=1 / N, \quad h=\pi / M, \quad t_{k}=k \tau, \quad k=-N, \ldots, N, \\
x_{n}=n h, \quad n=0, \ldots, M, \\
u_{n}^{0}=u_{n}^{1}=0, \quad n=0, \ldots, M, \quad u_{0}^{k}=u_{M}^{k}=0, \quad k=-N, \ldots, N .
\end{array}\right.
$$

Similarly, applying the second order of accuracy difference scheme (6) to the problem (19), we have

$$
\left\{\begin{array}{l}
\frac{u_{n}^{k+1}-2 u_{n}^{k}+u_{n}^{k-1}}{\tau^{2}}-\frac{u_{n+1}^{k}-2 u_{n}^{k}+u_{n-1}^{k}}{2 h^{2}}-\frac{u_{n+1}^{k+1}-2 u_{n}^{k+1}+u_{n-1}^{k+1}}{4 h^{2}}-\frac{u_{n+1}^{k-1}-2 u_{n}^{k-1}+u_{n-1}^{k-1}}{4 h^{2}} \\
=\left(\frac{2 t_{k}^{3}}{3}+t_{k}^{2}+2\right) \sin x_{n}+\tau \sum_{i=-k+1}^{k}\left(\frac{u_{n+1}^{i}-2 u_{n}^{i}+u_{n-1}^{i}}{2 h^{2}}+\frac{u_{n+1}^{i-1}-2 u_{n}^{i-1}+u_{n-1}^{i-1}}{2 h^{2}}\right) \\
\quad n=1, \ldots, M-1, k=1, \ldots, N-1, \\
\frac{u_{n}^{k+1}-2 u_{n}^{k}+u_{n}^{k-1}}{\tau^{2}}-\frac{u_{n+1}^{k}-2 u_{n}^{k}+u_{n-1}^{k}}{2 h^{2}}-\frac{u_{n+1}^{k+1}-2 u_{n}^{k+1}+u_{n-1}^{k+1}}{4 h^{2}}-\frac{u_{n+1}^{k-1}-2 u_{n}^{k-1}+u_{n-1}^{k-1}}{4 h^{2}} \\
=\left(\frac{2 t_{k}^{3}}{3}+t_{k}^{2}+2\right) \sin x_{n}-\tau \sum_{i=k+1}^{-k}\left(\frac{u_{n+1}^{i}-2 u_{n}^{i}+u_{n-1}^{i}}{2 h^{2}}+\frac{u_{n+1}^{i-2}-2 u_{n}^{i-1}+u_{n-1}^{i-1}}{2 h^{2}}\right) \\
\quad n=1, \ldots, M-1, k=-N+1, \ldots,-1, \\
\tau=1 / N, \quad h=\pi / M, \quad t_{k}=k \tau, \quad k=-N, \ldots, N, \\
x_{n}=n h, \quad n=0, \ldots, M, \\
u_{n}^{0}=0, \quad u_{n}^{1}=u_{n}^{-1}=\tau^{2} \sin x_{n}, \quad n=0, \ldots, M, \\
u_{0}^{k}=u_{M}^{k}=0, \quad k=-N, \ldots, N .
\end{array}\right.
$$

The difference schemes (20) and (21) are implemented by using the Gauss Elimination Method in Matlab. The errors are computed by

$$
E=\max _{\substack{-N \leq k \leq N \\ 0 \leq n \leq M}}\left|u\left(t_{k}, x_{n}\right)-u_{n}^{k}\right|
$$

where $u_{n}^{k}$ represents the numerical solution of the difference schemes at $\left(t_{k}, x_{n}\right)$. Table 1 shows the errors between the exact solution and the numerical solutions computed by using the first order and the second order of accuracy difference schemes (20) and (21), respectively. Table 1 is constructed using numerical solutions of the difference schemes for different values of $N$ and $M$. We observe that both schemes converge with the correct order. 


\section{Conclusion}

In this paper we have studied the second order of accuracy difference scheme approximately solving the initial value problem (1) for an integral-differential equation of the hyperbolic type in a Hilbert space $H$. The stability estimates for the solution of this difference scheme have been obtained. We have been able to confirm the correct order of the difference scheme by a numerical illustration for the simple test problem.

The aim of our future work is to apply high order of approximation two-step difference schemes [7-10] for an approximate solution of the initial value problem (1).

\section{Competing interests}

The authors declare that they have no competing interests.

\section{Authors' contributions}

Both authors contributed equally to this work. Both authors read and approved the final manuscript.

\section{Author details}

${ }^{1}$ Department of Mathematics, Fatih University, Buyukcekmece, Istanbul, 34500, Turkey. ${ }^{2}$ Department of Mathematics and Computer Sciences, Bahcesehir University, Besiktas, Istanbul, 34353, Turkey.

\section{Acknowledgements}

Authors are grateful to Prof. Dr. A. Ashyralyev (Fatih University, Turkey) for his comments and suggestions.

Received: 2 December 2013 Accepted: 3 April 2014 Published: 07 May 2014

\section{References}

1. Ashirov, S, Kurbanmamedov, N: Investigation of solution of one type of Volterra's integral equations. Izv. Vysš. Učebn. Zaved., Mat. 9, 3-8 (1987)

2. Ashirov, S, Mamedov, YD: A Volterra-type integral equation. Ukr. Mat. ž. 40(4), 510-515 (1988)

3. Krein, SG: Linear Differential Equations in a Banach Space. Nauka, Moscow (1966)

4. Ashyraliyev, M: A note on the stability of the integral-differential equation of the hyperbolic type in a Hilbert space. Numer. Funct. Anal. Optim. 29(7-8), 750-769 (2008)

5. Ashyralyev, A, Sobolevskii, PE: A note on the difference schemes for hyperbolic equations. Abstr. Appl. Anal. 6(2), 63-70 (2001)

6. Ashyraliyev, M: Generalizations of Gronwall's integral inequality and their discrete analogies, CWI Reports of MAS1, MAS-E0520 (2005). http://oai.cwi.nl/oai/asset/10927/10927D.pdf

7. Ashyralyev, A, Sobolevskii, PE: Two new approaches for construction of the high order of accuracy difference schemes for hyperbolic differential equations. Discrete Dyn. Nat. Soc. 2, 183-213 (2005)

8. Ashyralyev, A, Yildirim, O: On the numerical solution of hyperbolic IBVP with high order stable difference schemes. Bound. Value Probl. 2013, 29 (2013)

9. Ashyralyev, A, Sobolevskii, PE: New Difference Schemes for Partial Differential Equations. Birkhäuser, Basel (2004)

10. Sobolevskii, PE: Difference Methods for the Approximate Solutions of Differential Equations. Izdat. Voronezh Gosud University, Voronezh (1975)

\section{Submit your manuscript to a SpringerOpen ${ }^{\circ}$ journal and benefit from:}

$\rightarrow$ Convenient online submission

- Rigorous peer review

- Immediate publication on acceptance

- Open access: articles freely available online

- High visibility within the field

- Retaining the copyright to your article 